\title{
Relationship between optokinetic nystagmus response and recognition visual acuity
}

\begin{abstract}
Purpose The aim of this study was to investigate the relation between recognition visual acuity (RVA) and optokinetic nystagmus (OKN) response exhibited to different bar sizes with varying contrast. Methods OKN testing was performed in 52 children aged between 3 and 11 years. The children were evaluated in two groups according to their RVA. Group I consisted of 22 eyes with RVA equal to or better than 0.1 $\log$ MAR units. Group II consisted of 30 eyes with RVA 0.2-1 logMAR units. Each subject was seated $60 \mathrm{~cm}$ from the screen of Ophthimus ${ }^{\circledR}$ device, and was exposed to consecutive black and white stripes of seven different spatial frequencies (0.08-1.6 cycle/ degree). The narrowest bar that elicited OKN was identified, and then the OKN contrast threshold at this bar size was established. Results Twenty-one of the 22 eyes in Group I, and 26 of the 30 eyes in Group II exhibited 1.6 cycle/degree spatial frequency $(P=0.287)$. In Group II, $88.9 \%$ of the 18 eyes with RVA $0.2-0.5$ $\log M A R$ responded at this maximum spatial frequency, whereas the corresponding figure for the 12 eyes with RVA $0.6-1$ logMAR was $83.3 \%$ $(P=0.531)$. Contrast sensitivity (CS) significantly changed with age in Group $I(P=0.006)$. When the eyes that responded at maximum spatial frequency in the two groups were compared, the mean CS in Group II was significantly lower than that in Group I $(P=0.005)$.

Conclusions The results indicate no relation between spatial frequency threshold for OKN response and RVA in children. However, the children with RVA deficits had significantly lower CS.
\end{abstract}

Eye (2008) 22, 77-81; doi:10.1038/sj.eye.6702529; published online 11 August 2006
A Çetinkaya, S Oto, A Akman and YA Akova

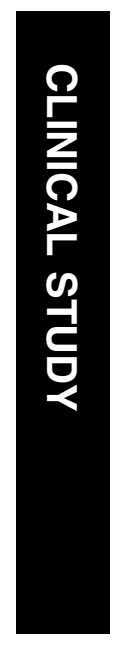

Keywords: contrast sensitivity; optokinetic nystagmus; recognition visual acuity

Introduction

Accurate visual testing of infants is very important. Early detection of visual acuity deficits in preverbal children would be valuable for ensuring that steps are taken to prevent amblyopia, and for diagnosing organic conditions that affect visual acuity. ${ }^{1,2}$ However, the conventional methods for evaluating infants' visual acuity in clinical settings have serious limitations. Three main techniques are currently used for objective assessment of visual function in children who are too young for standard subjective testing: (1) evaluation of optokinetic nystagmus (OKN) response,

(2) forced-choice preferential looking, and

(3) visual evoked potentials.,

$\mathrm{OKN}$ is the term for a series of reflexive eye movements that are observed even in newborns. ${ }^{5-7}$ The OKN response to stripes, which move across the individual's field of vision has been used as an objective way of measuring visual acuity for several decades. In this method of testing, the visual angle subtended by the smallest stripe width that still elicits an eye movement is taken as a measure of visual acuity. ${ }^{8}$ The OKN response was initially used by $\mathrm{Ohm}^{9}$ in an attempt to determine visual acuity, and a study by Gorman et $a l^{6}$ in 1957 is credited as having laid the foundation for modern measurements of visual acuity in infants.

Previous attempts have been made to correlate subjective and objective visual acuity by measuring the optokinetic visual acuity in an $\mathrm{OKN}$ apparatus, and then comparing this with
Department of

Ophthalmology, Başkent University Faculty of Medicine, Ankara, Turkey

Correspondence: S Oto, Hastanesi, Göz Hastalıkları Anabilim Dalı, Bahçelievler, Ankara 06490, Turkey Fax: +90 3122237333 E-mail: motility2000@ hotmail.com

Received: 30 January 2006 Accepted in revised form: 23 June 2006 Published online: 11 August 2006

Presented partly at the 28th Annual Meeting of European Strabismological Association, Bergen, Norway, 18-21 June 2003

The authors have no the equipments mentioned in this paper Başkent Üniversitesi Tel: + 90312 2156713; proprietary interest in any of 
standard Snellen visual acuity findings. ${ }^{10-15}$ Today's modern computer-assisted devices allow fine-tuning of both the contrast and the spatial dimensions of the stimulus presented. We wondered whether the new technology would enable more accurate clinical assessment of visual function in preverbal children, considering recognition visual acuity (RVA) levels as the gold standard. As it would be impractical to obtain RVA measurements using traditional distant VA charts (Snellen) under 3 years of age, we established our study group with children aged between 3 and 11 years. The specific aims of this study were to investigate whether OKN response can be quantified by varying spatial frequency and contrast, and to assess whether visual acuity based on OKN response shows a relationship with RVA levels. For these purposes, the lowest contrast level at the highest frequency was detected and these measurements were compared with each subject's RVA.

\section{Materials and methods}

Participants included 52 consecutive children, aged between 3 and 11 years, who appeared for refractive evaluation. Excluded from participation were patients with associated ocular anomalies, systemic disease, or neurological disorders. This research protocol was approved by the Institutional Review Board-Human Research Ethics Committee of the Başkent University. The purpose of the study has been explained in detail to each parent and informed consent was obtained before testing. Patients were evaluated in two groups. Group I consisted of 22 healthy children (age range, 4-10 years) with uncorrected RVA $0.1 \log$ MAR units or better. Group II consisted of 30 children (age range, 3-11 years) with uncorrected RVA 0.2-1.0 $\log$ MAR units. Only the right eye measurements were used for analysis. Group II was further divided into two subgroups depending on RVA for intra- and intergroup comparison purposes. Subgroup IIa consisted of 18 eyes with RVA 0.2-0.5 $\operatorname{logMAR}$, and subgroup IIb consisted of 12 eyes with RVA 0.6-1.0 logMAR.

The refractive errors were detected by using a calibrated autokerato-refractometer (Topcon, KR-7000, The Netherlands). The mean of five consecutive readings was recorded as the refractive error for the subject. Eyes with astigmatism measurements of $\geqslant 2$ diopters or $\geqslant 3 \times$ spheric values were considered to have astigmatic refractive error. The rest of the subjects were grouped as myopic or hyperopic according to the sign of spherical equivalent values. Children with visual loss owing to reasons other than refractive error were excluded from the study.

The right eyes were tested first with the left eye patched for both RVA and OKN measurements for every subject. RVA was evaluated using Snellen charts (letters, numbers) at $6 \mathrm{~m}$ or 'Lea Hyvärinen' charts at $3 \mathrm{~m}$, based on each child's capabilities. The last line that the child could recognize at least three out of five letters/figures was recorded as the visual acuity level. When the child recognized less than three characters, then the next lower line was accepted as RVA level. All RVA measurements were converted to logMAR values for statistical evaluation. The data from the right eyes were used for analysis in order to disregard the effect of fatigue observed in most of the children, especially at younger age (left eyes were tested after the right ones). OKN was elicited using a standardized OKN stimulator program in a computerized Ophthimus ${ }^{\circledR}$ device (Version 3.0, High Tech Vision, Göteborg, Sweden). The computer generates drifting square-wave luminance patterns of seven different spatial frequencies presented on a 15 inch NEC Multi-Sync XV15 colour monitor with a frame rate of $60 \mathrm{~Hz}$. The contrast level was available for 30 different steps in the seven grades of spatial frequency. The 1-unit increments ranging from 30 units to 1 unit represented from 97.5 to $3 \%$ contrast. With a child seated $60 \mathrm{~cm}$ away, the screen subtended a visual angle of $23.4 \times 19.5^{\circ}$.

The speed and spatial frequency values that corresponded to each bar size used in the test are shown in Table 1.

The luminance of the black and white stripes and the contrast levels represented by some of the contrast units used in the test are shown in Table 2. Michelson formula (Imax-Imin/Imax + Imin) was used to define the contrast level (\%), and the term contrast sensitivity (CS) stands for the inverse of the contrast level (1/contrast).

The brightness of the test room was measured adjacent to each subject's eyes using a luminance meter (Hagner ED1, Sweden) directed towards the computer screen, and was adjusted to $10 \mathrm{~cd} / \mathrm{m}^{2}$ before each test. The test was started after the child was seated on a comfortable, adjustable chair $60 \mathrm{~cm}$ from the screen with his/her eye level at the middle of the screen. If the child would not stay in this position, he or she was placed in a parent's

Table 1 Speed and spatial frequency values for the seven bar sizes that were used for testing optokinetic nystagmus response at $60 \mathrm{~cm}$ testing distance

\begin{tabular}{llcc}
\hline $\begin{array}{l}\text { Bar size } \\
\text { (unit) }\end{array}$ & $\begin{array}{c}\text { Bar width } \\
(\mathrm{cm})\end{array}$ & $\begin{array}{c}\text { Speed } \\
\text { (cycles/s) }\end{array}$ & $\begin{array}{c}\text { Spatial frequency } \\
\text { (cycles/degree) }\end{array}$ \\
\hline 7 & 6.5 & 0.76 & 0.08 \\
6 & 3.25 & 1.53 & 0.16 \\
5 & 2.6 & 1.9 & 0.20 \\
4 & 1.625 & 3.0 & 0.32 \\
3 & 1.3 & 3.8 & 0.40 \\
2 & 0.65 & 7.6 & 0.80 \\
1 & 0.325 & 15 & 1.6 \\
\hline
\end{tabular}


Table 2 Luminance of the black and white stripes, and their contrast level and contrast sensitivity equivalents represented by the contrast units used for optokinetic nystagmus testing

\begin{tabular}{lcccc}
\hline $\begin{array}{l}\text { Contrast } \\
\text { (units })\end{array}$ & $\begin{array}{c}\text { Black bar } \\
\left(\mathrm{cd} / \mathrm{m}^{2}\right)\end{array}$ & $\begin{array}{c}\text { White bar } \\
\left(\mathrm{cd} / \mathrm{m}^{2}\right)\end{array}$ & $\begin{array}{c}\text { Contrast } \\
\text { level }(\%)\end{array}$ & CS \\
\hline 30 & $<1$ & 80 & 97.5 & 1.03 \\
25 & 5 & 70 & 87 & 1.15 \\
20 & 10 & 60 & 71 & 1.4 \\
15 & 15 & 50 & 54 & 1.86 \\
10 & 20 & 40 & 33 & 3.0 \\
5 & 23 & 35 & 21 & 4.83 \\
3 & 27 & 33 & 10 & 10 \\
1 & 29 & 31 & 3 & 30 \\
\hline
\end{tabular}

lap and the parent helped hold the head in position if necessary. The child was asked to look at the centre of the screen and focus on the stripes. For the younger children who tended to tire before the test was complete, we held up transparent paper with drawings in front of the screen in order to keep their attention focused.

The test was started using the widest bar size (bar \#7) and the highest contrast level (30 units $=97.5 \%$ contrast). Each subject was tested monocularly and without corrective glasses. Two examiners were in the test room: one stayed behind the screen looking at the child's face from the front, and the other (who also set the computer parameters) stayed at an angle that allowed him to see both the child's face and the computer screen. When the two testers agreed that they had observed at least three consecutive beats of OKN, the width of the bar was scored as a pass. Stimuli were presented for as long as the observers needed to make a judgment (at least $20 \mathrm{~s}$ ). The smallest bar size that elicited OKN was identified, and the corresponding spatial frequency was recorded. Next, the contrast threshold at this spatial frequency was determined in a stepwise manner by decreasing the contrast level in five-unit increments until no response was elicited, and then increasing again in one-unit increments until the OKN response was detected again. The stimulus was presented in both nasal and temporal directions at the maximum spatial frequency level. Observation of OKN response was preferred instead of OKN recording by instruments because we aimed to evaluate the OKN response method as a simple, practical clinical assessment. However, placing electrodes is both time consuming for the tester and frightening for the young children, therefore impractical in either way.

\section{Statistical analysis}

The age distributions in the two groups were compared using the Student's $t$-test. The RVA levels in three refractive error subgroups of Group II were compared using analysis of variance (ANOVA). Fisher's exact test was used to compare the proportions of achieved spatial frequency levels in Groups I and II and to compare the distribution of achieved spatial frequency levels in relation to RVA between Groups IIa and IIb. KruskalWallis test was used to assess the relationship between mean spatial frequency level and type of refractive error in Group II. The mean contrast levels measured in different age groups in Group I were compared by Kruskal-Wallis ANOVA. Finally, the mean contrast levels of children exhibiting spatial frequency of 1.6 cycles / degree in the two groups were compared using the two-tailed $t$-test.

\section{Results}

The age distributions in Groups I and II were similar $(P=0.195)$. None of the 52 eyes exhibited asymmetrical nystagmus at the maximum spatial frequency level.

In Group I, four (18.2\%) of the 22 eyes had RVA of 0.1 $\log$ MAR and 18 had RVA 0 logMAR. The mean RVA in this group was $0.018 \log$ MAR $(S D=0.04)$. The mean RVA of the 30 eyes in Group II was $0.56 \log$ MAR $(S D=0.22)$. The distribution of refractive error and RVA in this group is shown in Table 3. There was no statistically significant difference between the RVA levels of different refractive error types $(P=0.121)$. The hyperopic eyes showed the best mean RVA among the three refractive error groups.

Only one eye $(4.5 \%)$ of a 4-year-old in Group I exhibited an OKN response at spatial frequency 0.8 cycles/degree (bar \#2), but the remaining 21 eyes all responded at the maximum possible frequency (bar $\# 1=1.6$ cycles/degree). In Group II, $26(86.7 \%)$ of the 30 eyes showed an OKN response at the maximum spatial frequency, and four eyes responded at spatial frequency 0.8 cycles/degree (bar \#2). Comparison of the

Table 3 Distribution of RE and RVA in Group II (30 eyes total)

\begin{tabular}{lclll}
\hline$R E$ & No of eyes & Range of RE $(S E)$ & Mean RVA $(\log M A R) \pm S D$ & Mean $S E \pm S D$ \\
\hline Myopia & 5 & $-1.0 ;-14.0$ & $0.72 \pm 0.22$ (range: 0.4-1.0) & $-4.2 \pm 5.52$ \\
Astigmatism & 17 & $-2.0 ;+3.50$ & $0.57 \pm 1.9$ (range: 0.3-1.0) & $0.07 \pm 1.50$ \\
Hyperopia & 8 & $+1.75 ;+6.50$ & $0.46 \pm 0.25$ (range: 0.2-0.8) & $3.34 \pm 1.49$ \\
\hline
\end{tabular}

$\mathrm{RE}=$ refractive error; $\mathrm{RVA}=$ recognition visual acuity; $\mathrm{SE}=$ spherical equivalent; $\mathrm{SD}=$ standard deviation. 


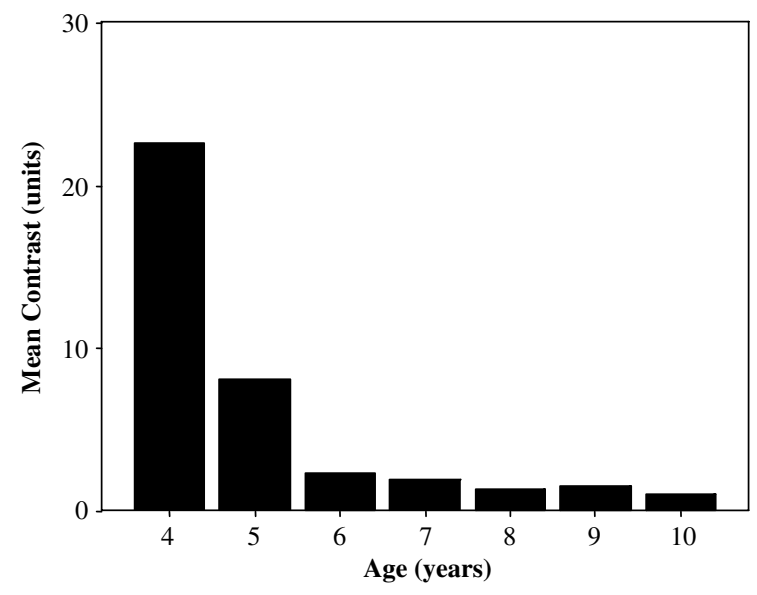

Figure 1 Distribution of ophthimus contrast units according to age in Group I.

proportion of eyes in each group that responded at maximum spatial frequency revealed no significant difference $(P=0.287)$.

To investigate the relationship between RVA and spatial frequency, we compared the spatial frequency findings in subgroup IIa (RVA 0.2-0.5 logMAR) and subgroup IIb (RVA 0.6-1.0 logMAR). Sixteen out of 18 eyes (88.9\%) in subgroup IIa and 10 out of 12 eyes (83.3\%) in subgroup IIb showed OKN at the maximum spatial frequency. There was no significant difference between these two proportions $(P=0.531)$. There was also no statistical significance of OKN response among different refractive error types. $(P=0.430)$. However, the fact that the hyperopic eyes tended to respond at lower spatial frequency even though they tended to exhibit better visual acuity may be of clinical importance.

Figure 1 shows the distribution of mean contrast (in units) level according to age in Group I. The increase in CS $(1 /$ contrast) in relation to increasing age was significant $(P=0.006)$. The mean CS in Group I was $23.8 \pm 11.66$ units, and the corresponding level in Group II was 13.04 \pm 13.2 units. As mentioned, the proportions of eyes in the two groups that responded at maximum spatial frequency were similar (21/22 in Group I and 26/30 in Group II). When the mean contrast levels for these two subsets of Group I and Group II eyes were compared, the level in Group II was significantly higher $(P=0.005)$.

\section{Discussion}

OKN is controlled by the nucleus of optic tract and dorsal terminal nucleus of the brain stem, and the middle temporal and middle superior temporal regions of the cerebral cortex, and does not require normal functioning of higher cognitive processes when the stimulus is visible. $^{16}$
The contrast sensitivity function (CSF) — obtained by measuring the CS for sine-wave grating of various spatial frequencies - has been proposed as a valuable addition to the psychophysical tests that measures acuity as well as other properties of form vision. ${ }^{7,16,17}$ CS measurement by $\mathrm{OKN}$ requires functioning afferent visual pathways, some amount of cortical and subcortical processing, and an efferent eye movement response.

Previous studies sought a possible relationship of OKN visual acuity with that of Snellen visual acuity to be used in clinical practice. Some authors have tried to estimate the visual acuity by relating the narrowest width of stripes, which elicits OKN response. ${ }^{12,13,18}$ Khan et al ${ }^{11}$ used the Catford visual acuity apparatus to determine the objective visual acuity. Where eyes with normal or near-normal vision showed good correlation between OKN response and visual acuity, no correlation was observed in eyes with poor vision and the authors concluded that objective methods of visual acuity testing using a nystagmoid response did not appear to be useful for general clinical purposes. This situation was further discussed in the study of Campos and Chiesi, ${ }^{3}$ who pointed to the interpretative pitfalls of the procedure declaring that the contrast resolution, which is required to obtain a Snellen visual acuity, was not the same phenomenon as the perception of vertical stripes moved on a horizontal plane. Besides emphasizing the effect of motion and the difference in the area of retina stimulated, they also mentioned about the technical difficulties in obtaining the narrow stripes to determine the threshold of spatial frequency and concluded that $\mathrm{OKN}$ cannot provide reliable qualitative information on the visual functions.

The effect of the contrast was also evaluated in various studies in addition to the bar size in OKN response. ${ }^{10-12,14,19}$ The visual acuity had decreased when filters were used in order to decrease the contrast; however, same kind of relation was not found when illumination was changed or when fogging lenses were used that had no influence on contrast. Gruber ${ }^{10}$ reported the correlating acuity levels of OKN to Snellen chart, using Bangerter's filters for contrast reduction on regular continuous OKN. Leguire et al ${ }^{19}$ measured the contrast thresholds for involuntary OKN with gratings of different spatial frequency and discovered that the OKN-CSF approximated the psychophysical-movement CS rather than the psychophysical-form CSF.

Nowadays, computer-assisted OKN testing devices, which provide the chance of changing the stripe width and contrast are available and have some advantages over the hand-held OKN drums. First of all, it is easier to fixate the child on the stimulus because the computer screen stimulates a larger visual field. This is extremely important because a negative response to OKN can be misinterpreting simply owing to a lack of attention to the 
stimulus, especially at young age. The computer-based system is also valuable for having uniform space-average luminance and uniform rate of movement. Furthermore, it provides smooth and exactly regular stripes on the screen.

According to the results of our study, the OKN responses obtained from high and low recognition acuity groups were not statistically different regarding the highest reached spatial frequency of the stimulus. This suggested that OKN acuity levels may not be related with recognition acuity levels, where the visual acuity levels are between 0 and $1 \log$ MAR units. However, when CS in the highest spatial frequency level was considered, a significant impairment of CS in relation to decreasing visual acuity was evident. The CS was also found to be closely related with age, which may on the other hand be owing to immature attention processes in younger age.

There was no statistical relation between the three refractive error types and the spatial frequency threshold as well. However, hypermetropes who had better mean $\log$ MAR acuity among the refractive-type groups showed the worst OKN response on testing. This seems to have some clinical value despite the nonsignificance on statistical analysis. The reason for this contradiction could be the testing distance at which the hypermetropic children would obviously have the least visual acuity at $\mathrm{OKN}$ testing distance $(60 \mathrm{~cm})$, whereas better uncorrected RVA at distant testing (3-6 m).

On the basis of these findings, it appears that measurement of OKN acuity with varying spatial frequency but a standard contrast may not probe fully the visual acuity deficits in preverbal children. However, the lowest contrast level detected at the highest spatial frequency seems to be in relation to RVA levels. In other words, we detected better CS in eyes with better RVA. In order to estimate the visual acuity level of the tested eye, it seems to be of better value to detect the CS after determining the highest spatial frequency level.

\section{References}

1 Dobson V, Teller DY, Belgum J. Visual acuity in human infants assessed with stationary stripes and phasealternated checkerboards. Vision Res 1978; 18: 1233-1238.
2 Jacobson SG, Mohindra I, Held R. Visual acuity in infants with ocular diseases. Am J Ophthalmol 1982; 93: 198-209.

3 Campos EC, Chiesi C. Critical analysis of visual function evaluating techniques in newborn babies. Int Ophthalmol 1985; 8: 25-31.

4 Hoyt CS. Objective techniques of visual acuity assessment in infancy. Aust NZ J Ophthalmol 1986; 14: 205-209.

5 Garbutt S, Harris CM. Abnormal vertical optokinetic nystagmus in infants and children. Br J Ophthalmol 2000; 84: 451-455.

6 Gorman JJ, Cogan DG, Gellis SS. An apparatus for grating the visual acuity of infants on the basis of optokinetic nystagmus. Pediatrics 1957; 19: 1088-1092.

7 Brown AM, Lindsey DT, McSweeney EM, Walter MM. Infant luminance and chromatic contrast sensitivity: optokinetic nystagmus data on 3-month-olds. Vision Res 1995; 35: 3145-3160.

8 Von Noorden GK, Campos EC. Examination of the Patient-I. In: Richard L (ed). Binocular Vision and Ocular Motility Theory and Management of Strabismus, 6th ed. Mosby Inc: Missouri, 2002,pp 158-167.

$9 \mathrm{Ohm}$ J. Use of moving signs in connection with optokinetic nystagmus for objective testing of visual acuity. Klin Monatsbl Augenheilkd 1952; 120: 144-150.

10 Gruber H. Decrease of visual acuity in patients with clear media and normal fundi: objective screening methods for differentiation and documentation. Doc Ophthalmol 1984; 56: 327-335.

11 Khan SG, Chen KFC, Frenkel M. Subjective and objective visual acuity testing techniques. Arch Ophthalmol 1976; 94: 2086-2091.

12 Millodot M, Miller D, Jernigan ME. Evaluation of an objective acuity device. Arch Ophthalmol 1973; 90: 449-452.

13 Reinecke RD, Cogan DG. Standardization of objective visual acuity measurements. Arch Ophthalmol 1958; 60: 418-421.

14 Catford GF, Olivier A. Development of acuity. Arch Dis Child 1973; 48: 47-50.

15 Wolin LR, Dillman A. Objective measurement of visual acuity. Arch Ophthalmol 1964; 71: 822.

16 Sakai S, Hirayama K, Iwasaki S, Yamadori A, Sato N, Ito A et al. Contrast sensitivity of patients with severe motor and intellectual disabilities and cerebral visual impairment. J Child Neurol 2002; 17: 731-737.

17 Abe H, Hasegawa S, Takagi M, Yoshizawa T, Usui T. Contrast sensitivity for the stationary and drifting vertical stripe patterns in patients with optic nerve disorders. Ophthalmologica 1993; 207: 100-105.

18 Enoch JM, Rabinowicz IM. Early surgery and visual correction of an infant born with unilateral eye lens opacity. Doc Ophthalmol 1976; 41: 371-382.

19 Leguire LE, Zaff BS, Freeman S, Rogers GL, Bremer DL, Wali N. Contrast sensitivity of optokinetic nystagmus. Vision Res 1991; 31: 89-97. 